\title{
Looking for logic
}

\author{
Improved tools and expanding knowledge are enabling new insights into the biochemical basis, \\ ecological roles and promising applications of natural product biosynthesis.
}

\begin{abstract}
Natural products have been knowingly intertwined with human experience for quite some time, whether within mixtures as traditional Chinese medicines, as extracted dyes for fabrics or as discrete antibiotics isolated from moldy bread. The utility of these compounds in human endeavors,
\end{abstract} coupled with scientists' intellectual curiosity, has propelled a quest for insights into their chemical, enzymatic and organismal origins. Recent advances in the creation or assimilation of innovative techniques have led to unprecedented opportunities to explore, define and exploit the logic of natural product biosynthesis. This themed issue provides a collection of articles that capture changing concepts and capabilities within the field.

At the heart of natural product biosynthesis are the enzymes-often encoded in biosynthetic gene clusters and expressed as multimodular assembliesthat link, modify and cut metabolic or peptidic precursors to create several classes of chemical architectures. As Walsh notes (Commentary, p. 620), decades of research in characterizing these catalysts has led to the identification of recurrent patterns, allowing the enumeration of rules in which the specificity of individual enzymes determines what building blocks will be incorporated and how they will be modified. When combined with the order of these enzymes within the overall assembly, this information can be used to rationalize or predict the resultant compounds.

Increasing numbers of genomes have provided additional templates on which to test these rules, and demonstrations that predictions can guide experimental energies in productive directions have provided a strong impetus for the development of improved informatics techniques and databases. Indeed, Medema and Fischbach (Perspective, p. 639) describe their vision of research in the field a decade from now as enabled by informatics, in which a gene cluster capable of making the desired analog of a particular molecule can be quickly identified from comparisons across thousands of genomes, the appropriate DNA synthesized and the molecule produced in rapid succession. To facilitate this kind of approach, a coalition of 150 scientists suggests that a new data standard is needed to make the reporting of biosynthetic genes more systematic (Commentary, p. 625). These authors further recommend that the new standard, termed 'MIBiG', become part of best practice for scientific publishing; to support this initiative, we will be asking authors to include MIBiG accession codes in reports of new biosynthetic gene clusters, and we ask referees to assist us by requesting and verifying the information provided.

Although informatics can now often predict with good success what molecule will come out of these enzymatic production lines, many questions still remain about how these systems operate in physical terms, in part because of their recalcitrance to traditional structural biology techniques. In addition to exploring the early challenges and successes in this area, Weissman (Review, p. 660) describes several recent structures-many enabled by the incorporation of techniques such as cryo-electron microscopy and small-angle $\mathrm{X}$-ray scattering into the methodological toolbox-that are transforming our understanding of intermodule interactions and allowing new questions to be posed regarding the processivity and specificity of the assembly lines.

Even as we obtain our first glimpses into the mechanical workings of known clusters, the continual flood of genomic data points to yet more clusters that can be explored in the search for new enzymes or assemblies that, in tandem, create unexpected chemical diversity. But how much diversity can we still expect to uncover? Walsh highlights several families of enzymes, such as oxidative enzymes and radical $S$-adenosylmethionine (SAM) enzymes, where each new sequence seems to harbor a surprise. Even enzymes that have been annotated as having unexceptional functions can yield unexpected outcomes when coupled to established pathways, such as the ability of a pyrophosphatase to counterintuitively boost natural product concentrations by dephosphorylating a substrate for terpene cyclases (Research Highlights, p. 633), or in unusual settings, such as the ability of a cytochrome $\mathrm{P} 450$-aldo-keto reductase fusion-but not the isolated enzymes-to isomerize reticuline (Article, p. 728). Walsh also notes that chemical diversity can readily be derived from modifications such as cyclization or prenylation to ribosomal and nonribosomal peptides and glycosylations of diverse natural products, both of which deserve further exploration.

Despite the breadth of architectural diversity available, the depth of structural analogs available for any one particular compound may be found to be limiting when that compound is deemed interesting for clinical, agricultural or other purposes. Almost as soon as the genetic basis for biosynthetic pathways was discovered, scientists began working to reengineer these pathways to produce molecular variations. Kim, Moore and Yoon (Perspective, p. 649) review some early successes and obstacles in the field of combinatorial biosynthesis and provide a vision for how synthetic biology tools can be employed to test hypotheses and speed solutions in this burgeoning area.

Biosynthetic gene clusters are more than miniature synthetic chemists: these operons also encode elements that speak to the timing and localization of compound production as well as the defense mechanisms the host uses to protect itself from these often toxic molecules. Keller (Review, p. 671) explores these issues in fungi, where transcriptional regulators work in tandem with transporters that traffic natural products, duplicated targets that can rescue a cell from natural products' inhibitory activity, and detoxification enzymes that can disarm reactive functional groups. Investigations of these understudied sequences can illuminate the natural function of natural products. For example, examination of a monooxygenase from a rice blast fungus identified its role in converting a fungally produced and excreted plant hormone into an alternate form to enable fungal infection (Article, p. 733).

Humanity's relationship with natural product biosynthesis has a long history, and recent insights-for example, in the identification of the enzymatic and organismal basis for production of bile acids in the gut (Article, p. 685)-have demonstrated that this relationship is far more complex and intimate than previously imagined. Our continued fascination with unraveling the biochemical rules and biological functions of these intriguing pathways is, therefore, only logical. 